\title{
Un nuevo modelo de innovación tecnológica del agro nicaragüense ${ }^{1}$
}

\author{
Manuel Enrique Pedroza Pacheco
}

Miembro de la Academia de Ciencias de Nicaragua

Parto del análisis sectorial del agro nicaragüense, en el cual existen serios problemas afectando el desarrollo del sector, tales como:

- Atraso tecnológico

- Baja productividad agrícola

- Bajo nivel de inversión y pobre disponibilidad de maquinaria y equipos

- Altos costos de producción

- Obsoleta infraestructura

- Poco desarrollo de los servicios de asistencia técnica

- Poco acceso al crédito y el disponible tiene altísimas tasas de interés

- Altos riesgos de inversión

- Inestabilidad en la propiedad

- Falta de valor agregado de los productos agrícolas-forestales

- Muchas áreas ociosas

- Baja coordinación interinstitucional en el sector agropecuario

¿Qué se puede hacer para transformar y superar esta realidad? No hay formulas mágicas para resolver una problemática institucional, tecnológica y socio-económica tan compleja como la del agro nicaragüense. Sin embargo, sin pensar que esto es lo único y lo concluyente, se propone: implementar una estrategia apropiada para la transformación productiva del agro nicaragüense que conlleve a: a) lograr el crecimiento del mercado de servicios tecnológicos vía oferta y demanda; b) crear más y mejores oportunidades de trabajo productivo, fundamentalmente en el sector rural; c) estimular la inversión nacional e internacional en Nicaragua, promoviendo la agroindustria y los agronegocios.

¿Cómo se puede cumplir ésta estrategia? Desarrollando una agricultura con conocimiento para la innovación tecnológica. Esto implica un amplio y profundo conocimiento de la "ruta crítica o camino largo" a seguir para lograr la innovación tecnológica en el campo. Así mismo, implica también conocer el "camino corto" a seguir y los factores obstaculizadores (incipiente desarrollo del mercado de tecnologías, baja adopción tecnológica, limitado acceso y disponibilidad de nuevas tecnologías en el campo, etc.) para lograr la innovación tecnológica en el agro nicaragüense. Para desarrollar una agricultura con conocimiento para la innovación tecnológica es necesario la aplicación de los siete enfoques contemporáneos de investigación, bases 
para desarrollar un modelo sistémico de innovación tecnológica del agro nicaragüense. Estos enfoques son:

1) Enfoque de Cadenas Productivas: Modelo I \& D.

2) Enfoque Sistémico: Redes; Pensamiento Sistémico; SI PS\&E; C I P P.

3) Enfoque de Competitividad: Entorno: Mercados; Clientes; Demanda ... FONTAGRO, FAITAN, RedSICTA, etc.

4) Enfoque Integrado de Investigación y Extensión en Sistemas Agropecuarios (IESA).

5) Enfoque de Desarrollo RuralIncluyente: Género, Participativo, Legitimidad, Compromiso, DEL; Territorialidad; Consistencia y Capacitación de Clientes, Beneficiarios, Socios, Usuarios.

6) Enfoque de Política Tecnológica.

7) Enfoque de Conservación y Manejo Sostenible de los Recursos Naturales.

De los siete enfoques, por brevedad, se destacan dos de ellos: Enfoque de Competitividad de Proyectos de I\&D, ya que existe un contexto de escaza disponibilidad de recursos financieros para la investigación e innovación tecnológica, así como una creciente demanda de clientes, beneficiarios, socios y donantes quienes reclaman satisfacer la demanda de nuevas tecnologías en el campo. Esto implica cambios importantes en la estructura de financiamiento para la innovación en el campo, cambios que están orientados hacia nuevos procedimientos y productos esperados, de acuerdo a nuevos objetivos y prioridades. Se destaca el enfoque IESA por ser una vía efectiva para la adopción y aplicación de nuevas tecnologías, esa es la clave de la innovación tecnológica.

Además de los siete enfoques contemporáneos de investigación, es muy importante profundizar en el concepto de Innovación Tecnológica, entendido como cambiar las cosas introduciendo novedades. La Innovación es una respuesta estratégica a la problemática de baja adopción tecnológica y baja productividad en el país. La innovación tecnológica, entendida de acuerdo al Manual de Oslo es el momento en el cual se verifica la apropiación social (vía mercado o no) de productos, servicios, procesos, métodos y sistemas que no existían anteriormente, o con alguna característica nueva y diferente de la vigente. La innovación, según el M.A. Mario López, implica la creación y aplicación de nuevas cosas y nuevo conocimiento cualitativamente diferente, innovación entendida como sinónimo de "nuevas combinaciones", y lo que se combina en la innovación son: diversas piezas de conocimientos y aprendizaje.

La innovación tiene una dimensión que trasciende a la I\&D, con una fuerte intersección en el proceso de adopción, por tanto va más allá de la investigación propiamente dicha, de modo que la innovación se realiza más en la esfera de la producción, comercialización, distribución, escalamiento, etc., que en la investigación. Son múltiples actores y múltiples perspectivas involucrados en los procesos de innovación. Dada la complejidad de actores y procesos está claro que el paradigma de GTTA que ha prevalecido en el agro nicaragüense en los últimos 40 años, hoy está transitando hacia el paradigma de innovación tecnológica. Este nuevo paradigma debe conducir tanto a la adopción de nuevas tecnologías, como a una mayor productividad y competitividad en el agro nicaragüense. Es necesario destacar que existen diferentes tipos de innovación: a) Innovación de procesos; b) Innovación de productos; y c) Innovación organizacional e institucional. 
La Innovación de productos: Como ejemplo se cita el maíz por sus múltiples usos y aplicaciones: El maíz tiene más de tres mil 500 usos y aplicaciones derivados de su compleja composición química. Del maíz se obtiene una gran diversidad de platillos alimenticios. La Maltodextrina, que es un subproducto derivado del maíz, se usa en la elaboración industrial del café expreso y del té. Cerca de 85 tipos diferentes de antibióticos utilizan maíz en sus fórmulas, como la capa fina que recubre las aspirinas y otros analgésicos, además de los sueros intravenosos y otros fármacos. Existen grandes avances en el área de fitomejoramiento en el cultivo del maíz en Nicaragua, 8 nuevas variedades en los últimos 15 años: NB-NUTRINTA, NUTRINTA-AMARILLO, INTA-OBATANPA, NUTRADER, NB-9043, HQ-INTA 993, HN-INTA 991, MAZORCA DE ORO.

La Innovación organizacional e institucional: En el contexto de Latino América y el Caribe: el gran reto es la transición de la generación y transferencia a la innovación tecnológica. La pregunta es: ¿qué tan innovadora es nuestra agricultura en ALC? El reto es: Desarrollar una agricultura con conocimiento, para lo cual una respuesta es promover innovaciones tecnológicas e innovaciones institucionales. En el contexto de Centro América, los desafíos son tres básicamente:

1. Estandarizar el concepto de innovación entre los miembros de SICTA.

2. Lograr el fortalecimiento de los INIAS, SNITAS y SICTA.

3. Incluir en la agenda de SICTA la agricultura familiar y el concepto de innovación social integral.

Los retos de la innovación institucional, en el contexto actual del sector agropecuario nicaragüense, a nivel macro institucional son: 1) Fortalecer el PPA, 2) Implementar la estrategia de PAIESA, 3) Fortalecer las alianzas estratégicas con universidades, 4) Desarrollar la unidad de adopción e innovación tecnológica, 5) Desarrollar los consejos tecnológicos y redes temáticas de innovación en el campo, 6) Implementar el FONICYT (Fondo Nicaragüense de Ciencia y Tecnología), y 7) Desarrollar la unidad de semillas, con visión multidisciplinaria, multisectorial e interinstitucional, en y para el contexto de nación.

Los retos de la innovación institucional, en el contexto actual del sector agropecuario nicaragüense a nivel meso institucional son: 1) Institucionalizar un nuevo modelo de innovación tecnológica del agro nicaragüense, 2) Desarrollar los CDT (Centros de Desarrollo Tecnológico), 3) Desarrollar un sistema integrado de planeación, seguimiento y evaluación, 4) Desarrollar el FINNTNIC (Fondo de Innovación Tecnológica de Nicaragua), 5) Crear el movimiento de promotores/innovadores agropecuarios, vinculados a los CDT, 6) Apoyar y desarrollar las capacidades gerenciales para la innovación en cada una de las oficinas del INTA, a nivel nacional y a nivel de cada territorio, y 7) Desarrollar el laboratorio nacional de Biotecnología.

Los retos de la innovación institucional, en el contexto actual del sector agropecuario nicaragüense a nivel micro institucional son: 1) Desarrollar el PRONINNT ${ }^{2}$ de Granos Básicos, 2) Desarrollar el PRONINNT de Producción Pecuaria, 3) Desarrollar el PRONINNT de Hortalizas y Frutas, 4) Desarrollar el PRONINNT de Raíces y Tubérculos, 5) Desarrollar el PRONINNT de Conservación y Manejo Sostenible de Recursos Naturales, 6) Desarrollar 
el PRONINNT de Agricultura Familiar, 7) Desarrollar el PRONINNT de Procesamiento de Alimentos.

Para estar a la altura de estos retos es necesario pasar de un modelo lineal de innovación e ir hacia un modelo sistémico de innovación. Por tanto se hace necesario implementar un nuevo modelo de innovación tecnológica del agro nicaragüense basado en los siete enfoques contemporáneos de investigación. Este nuevo modelo de innovación tecnológica debe tener características de: no lineal, sistémico, multidimensional, multidisciplinario, descentralizado, con diversidad y flexibilidad.

La Innovación de procesos: Como ejemplo se cita la estrategia PAIESA: un sistema de integración institucional que aglutina la pluralidad de actores y procesos de innovación tecnológica del nuevo INTA, universidades y cooperativas, etc., de modo que genere sinergia y complementariedad a lo interno y externo del INTA. La estrategia PAIESA promueve la coordinación horizontal, la mutua colaboración, los equipos multidisciplinarios de trabajo, la integralidad de actuación de los actores en los territorios, todo ello orientado a lograr una mayor adopción tecnológica e impacto económico en el campo.

Basado en la estrategia PAIESA, es la implementación, organización y desarrollo de los Consejos Tecnológicos, una nueva forma organizacional vinculante del nuevo INTA con los pequeños y medianos productores. Los productores son el eje central del trabajo del INTA, y constituyen la base subyacente del modelo de innovación tecnológica del agro nicaragüense, no sólo en la esfera propia de la investigación sino más allá de la investigación, la educación, la producción, la comercialización, el procesamiento y transformación de alimentos, esto es, trascender hacia la esfera de la producción, comercialización, distribución y escalamiento de los alimentos producidos.

Por tanto, el nuevo modelo promueve la integración sistémica de los diferentes actores que hacen los procesos de investigación-educación-producción en cada uno de los territorios. A través de los Centros de Desarrollo Tecnológico ocurre la innovación institucional que facilita la integración de los actores institucionales representados por organizaciones como MAGFOR, INTA, IDR, UNAG, INFOCOOP, UPANIC, APENN, universidades (UNA, UNAN, UCA, EIAG, UCATSE, URACCAN, BICU, UCC, etc.), alcaldías de cada municipio y asociaciones. Todos ellos, articulados con el sistema de educación primaria y secundaria, así como con el subsistema de educación agraria representado por el INATEC, constituyen parte importante del Sistema de Innovación Tecnológica Nicaragüense. Este es el significado de implementar el "Nuevo Modelo de Innovación Tecnológica del Agro Nicaragüense", base para mejorar el nivel y calidad de vida de la familia nicaragüense.

\section{Notas}

1 Modificado de la ponencia del autor en el Ciclo de Conferencias para la incorporación de los académicos de número de la Academia de Ciencias de Nicaragua, 17 de Marzo de 2010 en la Vicepresidencia de la República. PRONINNT: Programa Nacional de Innovación Tecnológica 